\title{
The Study of Flutter Speed Change Characteristics of Flat Wings After Trepanning
}

\author{
Duo Chen ${ }^{1, a}$, Li-Juan Chang ${ }^{2, b}$,
}

Dong Zhao ${ }^{3, \mathrm{c}}$, Ri-Li Hou ${ }^{4, \mathrm{~d}}$

${ }^{1}$ Department of civil Engineering, China Agriculture University , Beijing, 100083 China

${ }^{2}$ Chinese Academy of Building Research, Beijing, 100013 China

${ }^{3}$ China Nuclear Power Engineering Co, Beijing ,100840 China

${ }^{4}$ The First Aeronatic Institute of the Air Force ,Xinyang Henan.464000 China

a2270340548@qq.com, bzgndlingling@163.com, c394350818@qq.com,dhr16319@sina.com

Keywords: PK method, the finite element, trepanning about wings, flutter speed, MSC.Patran, MSC.Nastran

Abstract. There are many factors that cause the flutter of the wing of the aircraft, and one of the factors is the wing opening trepanning. In order to explore the changes of the flutter speed of the wing

after trepanning, the software simulation method is adopted to analyze the flutter speed .MSC.Patran and MSC.Nastran are used to model the structure of the wing structure, and the aerodynamic model is modeled by them. Then, the two part of the structural model is coupled, and modalities of the wing are obtained. Through the analysis of the calculation results, the change of the flutter speed of the plane flat wing is obtained, which provides a reference for the control of most subsonic airliners wings.

\section{Introduction}

Since the World War,the incident of plane crash internationally have continued. Some people in these accidents mainly died because of the aircraft flutter. Statistically, there were more than 100 kinds of flutter events in the United States. So a lot of methods on the airplane tremor analysis calculation for airplane flutter were created. For example, such as the sub sonic dipole grid method, the narrow strip theory, and the maher packet method, the supersonic dipole grid method, etc ${ }^{[1]}$... At the same time, a lot of softwares are born out for flutter analysis. But this does not mean that the flutter of the aircraft has been well sloved. Because the structure of the aircraft is diverse, and each structure has a different vibration, and each structure will have a different cause of the flutter ${ }^{[2]}$. This paper is devoted to the study of how the flutter speed of the aircraft change in the case of a trepanning flat wing. The size of the aperture will affect the stiffness of the wing. This paper will find out a factor which is only related to the size of the hole in the flat wing. It is necessary to study the control of a single variable.

\section{Basic theory}

To the calculation solutions of the wing flutter, there are a lot of different methods in the world, such as $\mathrm{K}$ method, KE method, PK method and so on. In this paper, the PK method is used to study destruction problems of the trpanning flat wings.PK method was proposed by a famous scholar named Hassigs ${ }^{[3]}$ in the early 1970s and then it was quoted in most fluttter calculations. Simplify the PK method and assump the wing do free movement,we can get :

$$
\left\{\begin{array}{l}
h=h_{0} e(\gamma+i) \omega t=h_{0} e^{p t} \\
a=a_{0} e(\gamma+i) \omega t=a_{0} e^{p t}
\end{array}\right.
$$


The vibration frequency:and from formula (2.1) it can introduce:

$$
r=\frac{1}{2 \pi} \ln \left[\frac{h\left(t+\frac{2 \pi}{\omega}\right)}{\mathrm{h}(\mathrm{t})}\right]
$$

$r$ indicates the vibration attenuation rate in formula(2.2).

In the critical flutter critical state, we can deem the wing to do simple harmonic vibration, and $\ddot{h}=-\omega^{2} h$, and the flutter equations are:

$$
\left[\frac{V^{2}}{b^{2}} M p^{2}+K+\frac{1}{2} \rho V^{2} A(K)\right]\left(\begin{array}{l}
\frac{h}{b} \\
a
\end{array}\right)=0
$$

In formula (2.3): $\mathrm{M}$ is the generalized inertia matrix, $\mathrm{K}$ is the generalized stiffness matrix, $\mathrm{A}$ is a generalized aerodynamic matrix, $\rho$ is the air density given height, $\mathrm{V}$ is the air speed, $\mathrm{B}$ is the half chord. So according to the frequency of the $\mathrm{k}=0$, the flutter speed can be determined after resloving the formula (2.3) .

\section{Dynamic modeling of finite element model of flat wing}

This study is based on the use of PK method,The MSC.Patran ${ }^{[4]}$ finite element software is used to model the structure dynamics of the flat wing,MSC.Patran can be used to finite element modeling of the structure, it also can be used to model the aerodynamic model at the some time, and coupling the two models,MSC.Nastran ${ }^{[5]}$ can be used to analyze and calculate the complex aerodynamic coupling models ,then it can get the damping $\mathrm{g}$ under different speeds.From this, the V-g diagram can be drawn out, the velocity is the speed of the flutter at $\mathrm{g}=0$.

\section{creating modeling}

In this paper,we study the characteristics of the flutter speed of the flat wing after trepanning,therefore, there are no special requiremenst and process for the model structure.So this study chooses a $1 \mathrm{~m} \times 1 \mathrm{~m}$ square plate, the thickness is $0.01 \mathrm{~m}$, the elastic modulus of the plate is $70 \mathrm{GPa}$, poisson's ratio is 0.3 , the material density is $2700 \mathrm{~kg} / \mathrm{m}^{3}$. The geometric center of the flat wing is cut into a circular plate of $0.05 \mathrm{~m}, 0.1 \mathrm{~m}$ and $0.2 \mathrm{~m}$ when analysing and calculating the openning hole model.To sum up, there are four kinds of models in this research: no openning hole model, hole radius in model is $0.05 \mathrm{~m}$, hole radius in model is $0.1 \mathrm{~m}$, hole radius in model is $0.2 \mathrm{~m}$. The structer model for calculation for the reseach is shown in figure 1 .

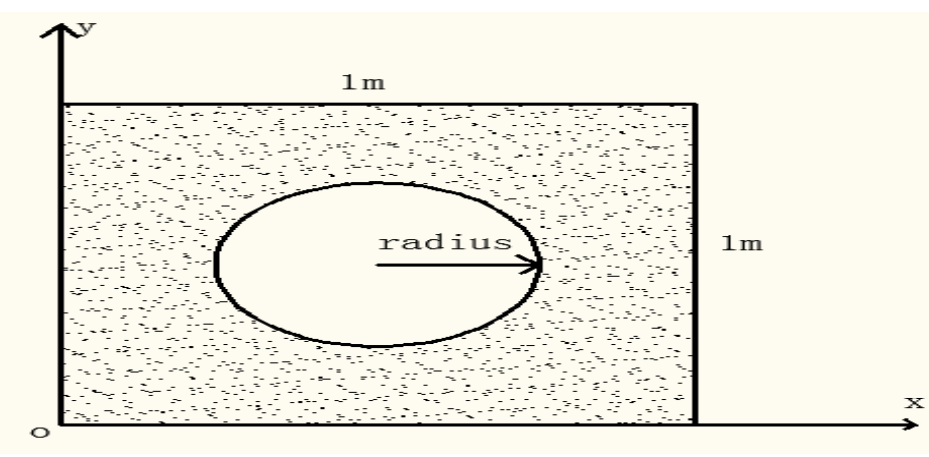

Fig. 1 The structer model for calculation

By using MSC.Patran software and MSC.Nastran to model and calculate, the third order torsional mode frequency for above four modelings are given by table 1 . 
Table 1.four mode frequency for four modelings

\begin{tabular}{lcccc}
\hline frequency & no hole model & hole radius $0.05 \mathrm{~m}$ & hole radius $\mathbf{0 . 1 \mathrm { m }}$ & hole radius $\mathbf{0 . 2}$ \\
\hline the third order & 48.566 & 48.914 & 48.728 & 47.716 \\
\hline torsional mode & & & & \\
\hline
\end{tabular}

At the same time, the third order torsional mode figures of above four modelings are given respectively by the following four figures.

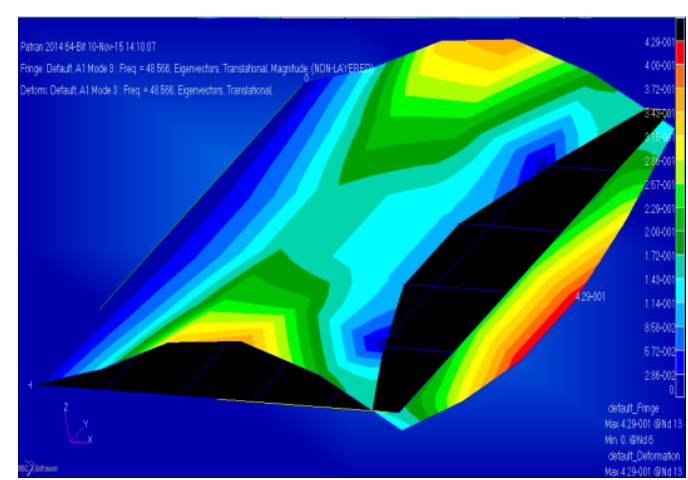

Fig. 2 The third order torsional mode figure of no hole radius of the model.

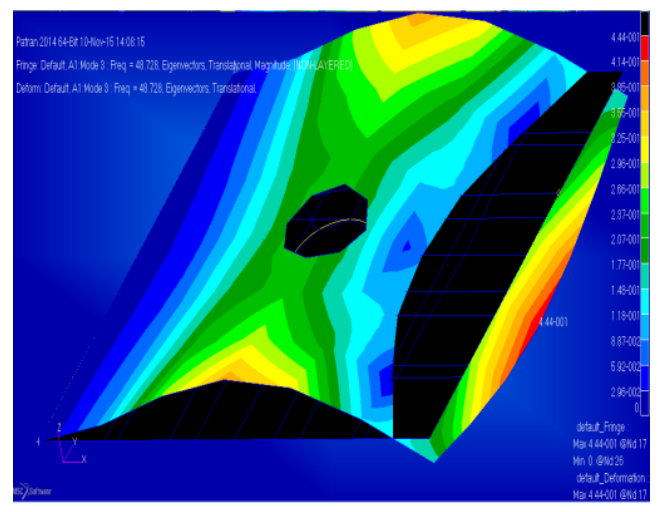

Fig.4 The third order torsional mode figure of $0.1 \mathrm{~m}$ hole radius of the model.

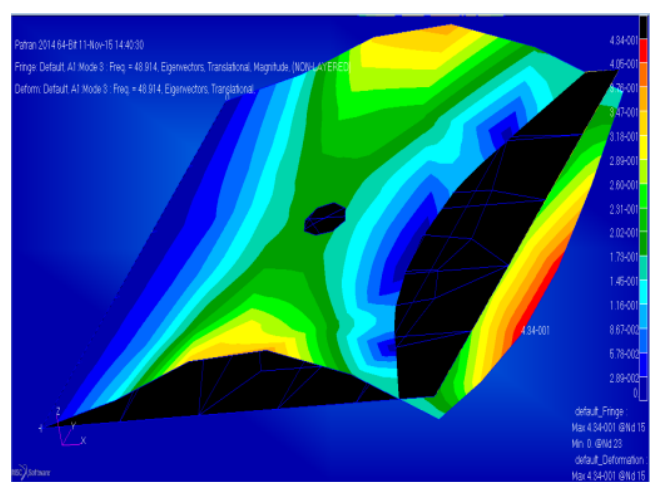

Fig.3 The third order torsional mode figure of $0.05 \mathrm{~m}$ hole radius of the model.

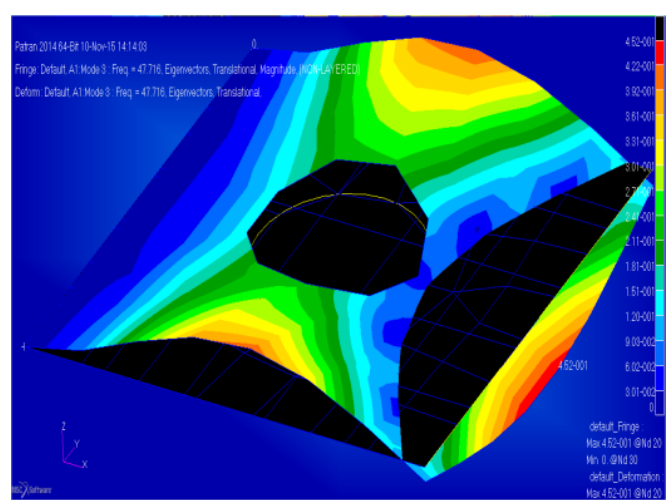

Fig. 5 The third order torsional mode figure of $0.2 \mathrm{~m}$ hole radius of the model.

By all above figures, the third order modalitys are respectively similar .Clearly the structure of the flat wing is changed, and the modal frequency is changed after the opening of the wing. This change is different with the different hole size, so the flutter speed of the wing will change with the same objective conditions.

\section{Analysis of the results in calculation}

When the structural aerodynamic coupling is calculated, the Maher number is 0.2 , the reduced frequency range is $0.1-0.2$, the air density is $1.2 \mathrm{~kg} / \mathrm{m} 3$, and the wing speed interval is $170 \mathrm{~m} / \mathrm{s}-220 / \mathrm{s}$, flight height can be set by MSC.softwares default, and assuming that the angle of attack of the wing is 1 degrees.On the basis of the above data, the four models are calculated respectively, and all the flutter speed of the wing is found in the third order mode. The flutter velocity at each model can be shown in Table 2 . 
Table 2.The flutter velocity at different models

\begin{tabular}{lcccc}
\hline & no hole & $0.05 \mathrm{~m}$ hole & $0.1 \mathrm{~m}$ hole & $0.2 \mathrm{~m}$ hole \\
\hline Velocity $(\mathrm{m} / \mathrm{s})$ & 215.45 & 213.40 & 198.54 & 171.55 \\
\hline
\end{tabular}

From the table 2 ,we can see: $\mathrm{V}_{1}=215.45 \mathrm{~m} / \mathrm{s}, \mathrm{V}_{2}=213.40 \mathrm{~m} / \mathrm{s}, \mathrm{V}_{3}=198.54 \mathrm{~m} / \mathrm{s}, \mathrm{V}_{4}=171.55 \mathrm{~m} / \mathrm{s}$.It is clear that the flutter speed relationship is $V_{1}>V_{2}>V_{3}>V_{4}$.In summary, the flutter speed of the flat wing destoryed is smaller, and it decreases with the increase of the hole diameter. Because of the size of the hole and the aperture of the structure, the stiffness of the structure will change the original flutter characteristics of the flat wing, which makes the flutter speed smaller. This is the same as the conclusion of the reference literature ${ }^{[6,7]}$, so the content and conclusions of this paper are set up.Also,

when the positions of the holes in the wing is different,the flutter velocity will be different with each other,and this discussion will not be mentioned in this paper.

\section{Conclusion}

The study proves that the effect about flutter of the structure of the aircraft wing after trepanning is notable.MSCpatran and MSCnastran make the analysis of aircraft wing's flutter characteristic easy, and it corresponds to reality with the actual analysis and calculation of the model and thus getting a reference value, which makes the aviation field of aircraft flutter analysis simple, especially reduces the original workload.The softwares provide the parameters that meet the requirements of various types of aircraft wings. Its finite element method and the calculation method are also greatly improved the computational accuracy. In this paper, the research flow of the change of the flutter speed of the flat wing is in accordance with the practical engineering significance. It can also be used in the characteristic research of the normal aircraft wings.

\section{Acknowledgement:}

Project supported by the National Natural Science Foundation of China (Nos. 11272340), the National Science and Technology Major Project(Nos. 2015ZX06004004-003)

Corresponding author Ri-Li Hou,E-mail:hr16319@ sina.com

\section{Referencs:}

[1] Zhao Yonghui. Gas dynamic elastic mechanics and control. Beijing[M]:Science Press,2007.

[2] Yi Chuanjia. Characteristics of an aircraft.Beijing[M]:Atomic Energy Press,2007.

[3] Hassig H J. An Approximate Ttue Damping Solution of the Flutter Equation by Determinant Iteration[J]. Journal of Aircraft,1978:8,pp.880-895.

[4] Liu Bingshan, Huang Cong. Patran from entry to the master[M]. Beijing:China Water Conservancy and Hydropower Press,2003.

[5] MSC.Flightloads and Aeroelastic Analysis User's Guide[G].CA:MSC Company Press,2001.

[6] Wang Nannan. Study on wing flutter and active control of two yuan.Beijing[D]:China University 
of Mining and Technology,2013.

[7] Wang Fei, He Jingwu.The bionic wing structure stiffness and strength design based on[J].Aircraft design press, 2010 . 